\title{
Resultado de Tratamientos Ortodóncicos y su Relación con la Complejidad de la Maloclusión
}

\author{
Outcome of Orthodontic Treatments and its Relationship to Malocclusion Complexity
}

\author{
Diana Maria Barbosa Lis*; Oscar Zapata Noreña*; Alvaro Carvajal*; Celba Maria Franco*; \\ Sara Astrid Rodriguez Aguirre ${ }^{* *}$; Alvaro Andres Florez Pino** \& Mariluz Restrepo**
}

\begin{abstract}
BARBOSA, L. D.; . D.; ZAPATA, N. O.; CARVAJAL, A.; FRANCO, C. M.; RODRIGUEZ, A. S. A.; FLOREZ, P. A. A. \& RESTREPO, M. Resultado de tratamientos ortodóncicos y su relación con la complejidad de la maloclusión. Int. J. Odontostomat., 8(2):201-206, 2014.
\end{abstract}

RESUMEN: Este estudio descriptivo evalúa los resultados clínicos estáticos de los tratamientos finalizados en el Posgrado de Ortodoncia de la Universidad de Antioquia durante el periodo 2010-2011 y su relación con la complejidad inicial y otros factores administrativos. A los pacientes terminados se les realizó un seguimiento documental de la historia clínica para determinar el grado de compromiso inicial y se evaluaron, con los parámetros del Objective Grading System (OGS) del American Board of Orthodontic (ABO), los modelos y las radiografías panorámicas finales. De 99 pacientes que terminaron el tratamiento, 40 cumplieron los criterios de inclusión. El $80 \%$ de los pacientes que terminaron el tratamiento tenían maloclusión comprometida o altamente comprometida al iniciar. El puntaje OGS del ABO encontrado fue en promedio de $31,7( \pm 8,5)$ puntos y el $50 \%$ de los casos pasaban los criterios del OGS. El tiempo de duración del tratamiento $(55 \pm 22,25$ meses) y el número de citas promedio para terminar el tratamiento $(37,3 \pm 11,4)$ aumentan según la complejidad de la maloclusión inicial. El grado de maloclusión inicial tuvo relación estadísticamente significativa con los puntajes de OGS $(p=0,018)$, mientras que otras variables administrativas y clínicas no afectaron los resultados. Los valores finales del OGS encontrados no son los ideales y son afectados por el compromiso de la maloclusión.

PALABRAS CLAVE: control de calidad, maloclusión, índice de necesidad de tratamiento, valoración de resultados.

\section{INTRODUCCIÓN}

El Posgrado de Ortodoncia de la Universidad de Antioquia forma recurso humano especializado en el tratamiento de las anomalías craneodentofaciales El mejoramiento continuo de la calidad es una política de estado y una herramienta para garantizar la correcta prestación de los servicios partiendo de la autoevaluación de los procesos. Aunque se ha investigado acerca de la satisfacción de los pacientes del posgrado de Ortodoncia de la Universidad de Antioquia y el 95,6\% están satisfechos con los resultados obtenidos (Hincapié et al., 2001), no existen publicaciones locales que evalúen la calidad de los resultados, la duración y la complejidad de los tratamientos de ortodoncia. Existe en el posgrado, evidencia documentada no publicada, sobre la duración prolongada de los tratamientos de ortodoncia y del bajo registro de pacientes terminados por año/por residente en el periodo 1995-2006 (1,5 pacientes terminados por estudiante).
Por ser un servicio de formación de alto nivel, en el posgrado se realizan tratamientos con diferentes grados de complejidad y este factor podría afectar el tiempo y la calidad de los resultados. Por esto se hace necesaria una revisión de la calidad de finalización de los tratamientos ortodóncicos, valoración que puede realizarse con diferentes herramientas empleadas en el ámbito formativo universitario e institucional en el mundo, entre ellas el Objective Grading System (OGS) propuesto por el American Board of Orthodontic (ABO) para evaluaciones estáticas de certificación de la calidad de la practica ortodóncica (Casko et al., 1998), que permita proponer planes de mejoramiento para corregir aspectos que puedan estar originando retardos y disminución en la calidad de los tratamientos, en la satisfacción de la población tratada y/o en el nivel de formación de los futuros ortodoncistas (YangPowers et al., 2002).

\footnotetext{
* Profesores Facultad de Odontología, Universidad de Antioquia, Medellín, Colombia.

**Facultad de Odontología, Universidad de Antioquia, Medellín, Colombia.
} 
El objetivo del estudio fue determinar los resultados clínicos estáticos de los tratamientos según el OGS del ABO, su relación con las características iniciales de la maloclusión y con otras variables administrativas, en los pacientes finalizados en el Posgrado de Ortodoncia de la Facultad de Odontología de la Universidad de Antioquia, en el periodo 2010-2011.

\section{MATERIAL Y MÉTODO}

Se realizó un estudio descriptivo-ambispectivo en 40 de 99 pacientes que finalizaron su tratamiento, que cumplieron los criterios de inclusión: pacientes que terminaron la fase activa de tratamiento de ortodoncia fija maxilo-mandibular entre los años 2010-2011, que iniciaron la fase de retención y aceptaron participar en la investigación.

Se excluyeron los pacientes que no aceptaron participar en el estudio (9), que continuaron con tratamientos protésicos o periodontales (46) o que presentaron ayudas diagnosticas inadecuadas (4). Cuarenta pacientes conformaron la muestra (21 mujeres y 19 hombres), a quienes se les evaluó la complejidad oclusal en su historia clínica, se les tomaron radiografías panorámicas y modelos de estudio inmediatamente después que se les retiró la aparatología.

Se realizó un análisis estático en los modelos de estudio y en las radiografías panorámicas, aplicando los criterios del OGS, método estandarizado para evaluar las características de los tratamientos ortodóncicos terminados, desarrollado por el $\mathrm{ABO}$, organismo internacionalmente reconocido como autoridad en la materia (Cameron, 2010; Casko et al.). Los criterios OGS son: alineación, rebordes marginales, inclinación buco-lingual, relaciones oclusales, contactos oclusales, sobremordida horizontal, contactos interproximales y angulación de la raíz. Se digitalizaron los modelos en la empresa IMAX (Imágenes Maxilofaciales S.A. Medellín, Colombia) con el fin de disminuir el grado de error y aumentar la confiabilidad de las medidas (Costalos et al., 2005; Paredes et al.,
2006; Hou et al., 2006); empleando el escáner Laser Ortho Insight 3D y el software Motion View Software Design LLC. Se realizaron pruebas piloto para validar el software y estandarizar el operador; la medida de los rebordes marginales no fue confiable, por lo que se empleó el instrumento tipo regla diseñado para ello por el $\mathrm{ABO}$ (Fig. 1); se construyeron 3 reglas con las medidas del ABO y se calibraron en Mebí (Metrología Biomédica, Medellín, Colombia) con un nivel de confianza del $95 \%$. Dos de los investigadores midieron la discrepancia de los rebordes marginales, previa calibración intra e inter-examinador hasta alcanzar un índice Kappa de 0,97. El criterio paralelismo radicular dental fue valorado, según el OGS, en las radiografías panorámicas, con calibración intra e inter-examinador, obteniéndose un índice Kappa de 0,98.

Siguiendo la guía del OGS (American Board of Orthodontics, 2009; Cangialosi et al.), la valoración en modelos y radiografías se inicia en 0 para cada paciente y se le suman los puntos correspondientes a las deficiencias encontradas en cada parámetro evaluado. Los puntajes de 20 o menos (excelentes) y entre 20 y 30 (aceptables) superan el OGS del ABO, los puntajes superiores a 30 (menos que aceptables), no pasan el OGS, ya que a mayor puntuación obtenida por el caso, menor cumplimiento de los parámetros (Casko et al.).

El grado de complejidad oclusal inicial de los pacientes se evaluó en la historia clínica, por ausencia de ayudas diagnosticas iniciales, creándose un índice de complejidad según el número de campos afectados (Ackerman \& Proffit, 1969) que presentaba el paciente (transversal, vertical, sagital, alineación y otros, otros se refiere a la presencia de situaciones como dientes supernumerarios, transposiciones, etc.) Si se afectaban hasta dos campos era levemente comprometido, tres campos era comprometido y hasta 5 campos era altamente comprometido. A un paciente no se le realizó el análisis de complejidad por carecer de información en su historia clínica. Adicionalmente se evaluaron aspectos administrativos como tiempo de tratamiento en meses, numero de citas de atención y tipo de tratamiento (con y sin extracciones)

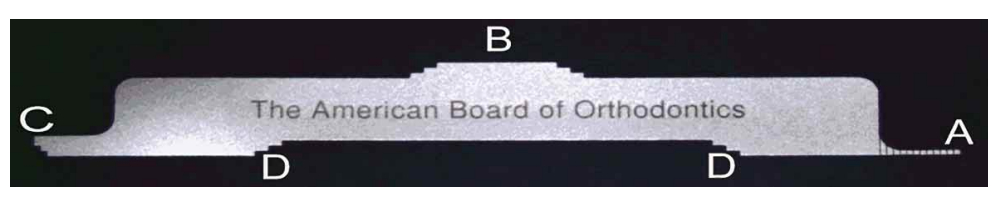

Fig.1. Instrumento desarrollado por la $A B O$ para medir modelos dentales, con divisiones de $1 \mathrm{~mm}$, la zona A mide las discrepancias en alineación, resalte, contacto oclusal, contacto interproximal y relaciones oclusales. La zona $\mathrm{B}$ mide la inclinación bucolingual mandibular. La zona $\mathrm{C}$ determina discrepancias en los rebordes marginales. La zona $\mathrm{D}$ se utiliza para la inclinación bucolingual maxilar. 
Aspectos éticos. Basados en la norma de Helsinki de 2008 (Asociación Médica Mundial, 2008) y en la resolución N008430 de 1993 (República de Colombia, Ministerio de Salud, 1993) de la República de Colombia, se establecieron los controles para garantizar la seguridad e informar los deberes, derechos y beneficios de los participantes en la investigación, de los responsables de su ejecución, de los procedimientos a realizarse y los actos de mitigación de cualquier hecho fortuito e inesperado que llegase a ocurrir.

Análisis estadístico. Se utilizó el programa estadístico SPSS versión 17 (SPSS Inc., Chicago, IL). Se utilizaron las frecuencias absolutas simples y relativas expresadas en porcentajes para describir el grado de complejidad inicial de los pacientes; se hizo análisis uni-variado para describir mediante promedios y desviaciones estándar las variables administrativas (tiempo de tratamiento y numero de citas) y análisis bi-variado con la prueba de ANOVA de una vía para evaluar si había o no diferencias entre las variables clínicas o administrativas respecto a: grado de complejidad, puntaje OGS y tiempo de tratamiento. Análisis multi-variado mediante regresión lineal múltiple para determinar que variables clínicas o administrativas tenían un peso significativo respecto al resultado final del OGS. Previo a este análisis se hicieron correlaciones bi-variadas mediante el coeficiente correlación de Pearson para identificar las variables clínicas y administrativas que tenían relación entre sí, para evitar el efecto de colinealidad. Valores $p \leq 0,05$ se establecieon como estadísticamente significativos.

\section{RESULTADOS}

Nivel de complejidad. El $80 \%$ de los pacientes tenían un grado de complejidad inicial entre comprometido y altamente comprometido, discriminados en el 28,2\% (11 pacientes) con 3 campos involucrados, el 43,6\% (17 pacientes) con 4 campos implicados y el 7,7\% (3 pacientes) con 5 campos involucrados (Fig. 2).

Complejidad y duración del tratamiento. Las variables administrativas como número de meses de tratamiento (promedio 55,5 $\pm 22,2$ ), número de meses efectivos de tratamiento (promedio $43,3 \pm 14,7$ ) y número de citas para terminar el tratamiento (promedio $37,3 \pm 11,4)$ tuvieron un comportamiento ascendente acorde a la complejidad inicial de la maloclusión (Fig. 3 ), sin diferencias significativas entre los meses de tratamiento para los pacientes comprometidos y los alta-

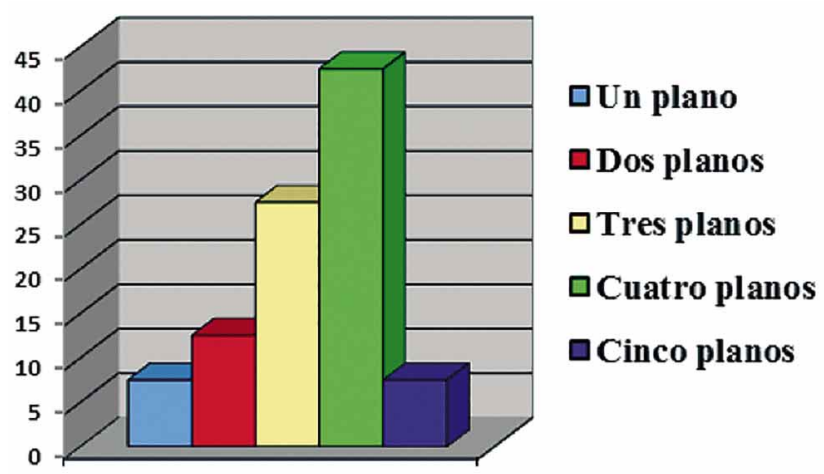

Fig. 2. Nivel de complejidad inicial de la maloclusión según los campos comprometidos.

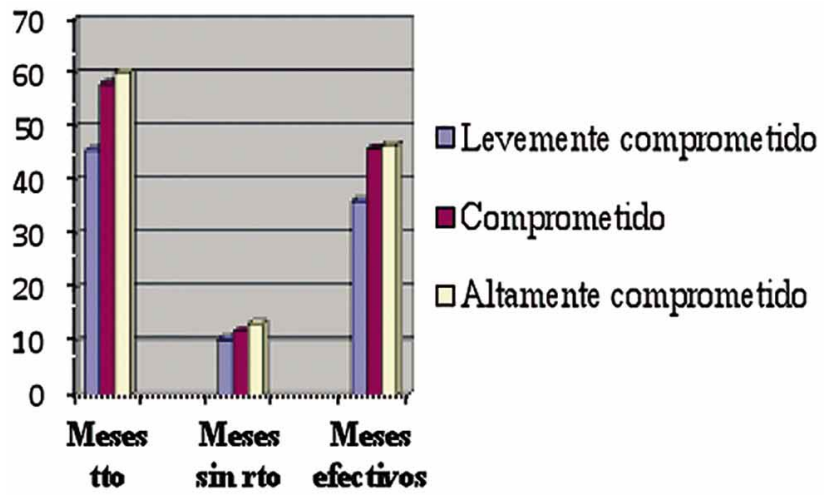

Fig. 3. Distribución de grado de complejidad y tiempo de tratamiento en los pacientes de la muestra.

mente comprometidos. Los meses sin registro en la historia clínica también tuvieron un comportamieno ascendente.

Complejidad y OGS del ABO. La evaluación del OGS estuvo entre 18 y 49 puntos (promedio $31,7 \pm 8,4$ ). Al comparar el OGS con el grado de complejidad inicial de la maloclusión se encontraron diferencias estadísticamente significativas $(p=0,018)$, con puntajes menores de OGS en los menos comprometidos y viceversa (Tabla I). Se observó un incremento gradual del OGS de 24,5 en los levemente comprometidos a 32,1 en los comprometidos y a 34,2 en los altamente comprometidos.

Al agrupar el puntaje OGS, cuatro pacientes tuvieron 20 puntos o menos (10\%), considerados excelentes, $40 \%$ aceptable, mientras que predominó el grupo de menos que aceptable con un $50 \%$. La Tabla II muestra la distribución del OGS agrupado según el grado de complejidad inicial.

EI $75 \%$ de los pacientes con OGS aceptable presentaban al comienzo del tratamiento un grado de com- 
BARBoSA, L. D.; . D.; ZAPATA, N. O.; CARVAJAL, A.; FRANCO, C. M.; RODRIGUEZ, A. S. A.; FLOREZ, P. A. A. \& RESTREPO, M. Resultado de tratamientos ortodóncicos y su relación con la complejidad de la maloclusión. Int. J. Odontostomat., 8(2):201-206, 2014.

Tabla I. Descripción y comparación del OGS según el grado de Complejidad.

\begin{tabular}{|c|c|c|c|c|c|}
\hline \multirow[b]{2}{*}{ Complejidad } & \multirow[b]{2}{*}{$\mathbf{n}$} & \multirow{2}{*}{$\begin{array}{c}\text { OGS } \\
\text { X } \pm \text { DE }\end{array}$} & \multicolumn{2}{|c|}{ IC $95 \%$} & \multirow{2}{*}{$\begin{array}{l}\text { ANOVA } \\
\text { Valor } p\end{array}$} \\
\hline & & & $\begin{array}{l}\text { Límite } \\
\text { inferior }\end{array}$ & $\begin{array}{l}\text { Límite } \\
\text { superior }\end{array}$ & \\
\hline Levemente comprometido & 8 & $24,5 \pm 5,42$ & 19,96 & 29,04 & \\
\hline Comprometido & 11 & $32.18 \pm 6,416$ & 27,87 & 36,49 & $0.018^{*}$ \\
\hline Altamente comprometido & 20 & $34,25 \pm 9,026$ & 30,01 & 38,49 & \\
\hline TOTAL & 39 & $31,67 \pm 8,471$ & 28,92 & 34,41 & \\
\hline
\end{tabular}

${ }^{*} p \leq 0,05$

Tabla II. Distribución de grado de complejidad según el OGS agrupado.

\begin{tabular}{lcccc}
\hline & \multicolumn{3}{c}{ Grado de compromiso } & \\
\cline { 2 - 4 } & $\begin{array}{c}\text { Levemente } \\
\text { comprometido }\end{array}$ & Comprometido & $\begin{array}{c}\text { Altamente } \\
\text { comprometido }\end{array}$ & TOTAL \\
\hline OGS (agrupado) & $\mathrm{n}(\%)$ & $\mathrm{n}(\%)$ & $\mathrm{n}(\%)$ & $\mathrm{n}(\%)$ \\
Excelente & $2(50 \%)$ & $0(0)$ & $2(50 \%)$ & $4(10 \%)$ \\
Aceptable & $5(31,3 \%)$ & $7(44 \%)$ & $4(25 \%)$ & $16(40 \%)$ \\
Menos que aceptable & $1(5 \%)$ & $4(20 \%)$ & $15(75 \%)$ & $20(50 \%)$ \\
TOTAL & $8(20 \%)$ & $11(27,5 \%)$ & $21(52,5 \%)$ & 40 \\
\hline
\end{tabular}

plejidad entre levemente comprometido y comprometido, mientras que de los terminados con OGS menos que aceptable, el $75 \%$ iniciaron el tratamiento con un grado de complejidad altamente comprometido. Llaman la atención dos pacientes altamente comprometidos al inicio, que terminaron con OGS excelente, un comportamiento diferente al resto de la muestra. No se encontraron diferencias estadísticamente significativas en el OGS en pacientes con y sin extracciones.

El análisis de regresión lineal múltiple no encontró ninguna variable administrativa estudiada que explicara el comportamiento del puntaje OGS.

\section{DISCUSION}

En este estudio se registraron 99 pacientes terminados en el periodo 2010-2011, de los cuales 40 cumplieron los criterios de inclusión. Este número de pacientes terminados es muy bajo para una institución que gradúa 12 residentes en 2 años. Sin embargo no se tienen datos publicados de los promedios de terminación de pacientes en otras instituciones universitarias colombianas o latinoamericanas. En la Universidad de Indiana (USA) (Pinskaya et al., 2004) se evaluaron los registros de 455 pacientes terminados en tres años consecutivos; en el estudio de Campbell (Campbell et al., 2007), se evaluaron 989 pacientes finalizados entre 1998 y 2003, en promedio 196 por año; mientras que Brown et al. (2011) reporto 714 pacientes terminados en la Universidad de Detroit en un periodo de 5 años, sin embargo estos estudios no reportan el número de residentes tratantes.
Complejidad. El logro de los objetivos del tratamiento ortodóncico puede variar según la severidad (complejidad) de la maloclusión inicial, cooperación del paciente, habilidad del operador y respuesta del paciente al tratamiento (Campbell et al.). En este estudio el $80 \%$ de los pacientes terminados tenían una complejidad inicial entre comprometidos y altamente comprometidos, similar a lo reportado por la Universidad de Indiana (Pinskaya et al.) donde el 68,3\% de sus paciente tenían un Índice de Discrepancia (ID) mayor de 10 o alto. Campbell (Campbell et al.) encontró un ID superior a 17, indicativo de una complejidad de moderada a alta. La Universidad de Detroit en el 2011 reportó gran variabilidad en el ID inicial de sus pacientes, pero todos con puntajes mayores de 10, lo cual sugiere un compromiso alto en su maloclusión (Brown et al.). La Universidad de Okayama reporto índices de complejidad alto para sus pacientes con un promedio de ID de 19,1 \pm 12 (Deguchi et al., 2005).

Se evidenció una relación positiva entre el OGS total y la complejidad inicial de la maloclusión $(p=0,018)$, indicando que a mayor complejidad más inadecuados los resultados finales del tratamiento. Esto es compatible con lo reportado por Vu et al. (2008) quien afirma que los pacientes con puntajes entre 10 y 20 del ID tenían resultados de tratamiento mejores que los pacientes que tenían ID superiores a 20. Campbell et al. encontró una correlación positiva entre el OGS y el ID, indicando que los pacientes con mayor ID eran más difíciles de finalizar y terminaban con OGS más altos al final del tratamiento.

Complejidad y duración de tratamiento. Se encontró que a medida que aumentaba la complejidad inicial del 
paciente, aumentaba el tiempo del tratamiento, acorde con lo reportado por Pinskaya et al., que halló una correlación estadísticamente significativa entre tiempo de tratamiento y altos puntajes en el OGS al finalizar. Vu et al. encontraron que el $45 \%$ de la variación en el tiempo del tratamiento podía explicarse por factores como el tipo de maloclusión y el ID, indicando que a mayor ID más tiempo para finalizar el tratamiento. Contrariamente, Saxe et al. (2010) no hallaron relación entre el nivel de dificultad y los resultados del tratamiento.

Complejidad y OGS de la ABO. Alford et al., (2011) evaluó los resultados finales usando dos técnicas ortodóncicas (Convencional y SurasmileR) y encontró un OGS promedio de 20,8 (mínimo 9 y máximo 38) con técnica convencional y 18,5 (mínimo 6 y máximo 38 ) con técnica SurasmileR. Stock et al. (2011) hallaron puntajes OGS de 14,7 para pacientes con los que usaron posicionador de finalización y de 23,0 con protocolo de finalización convencional. Campbell et al. determinaron OGS promedio de 32,64 en pacientes de la Universidad de Indiana. Brown et al. en la Universidad de Detroit obtuvo puntajes OGS entre 22,11 y 34,79 en 5 años consecutivos. En el presente estudio, el puntaje de OGS $(31,6 \pm 8,4)$ es alto sabiendo que mediciones mayores de 30 son menos que aceptables, sin embargo, este valor está dentro del promedio de los valores reportados para instituciones universitarias (Campbell et al.; Brown et al.; Alford et al.).

Otros factores que afectan al OGS son la ranura del bracket y la técnica utilizada (Alford et al.; Stock et al.). Detterline et al. (2010) encontró diferencias significativas utilizando ranuras $0,018(26,3 \pm 10)$ y ranuras $0,022(28,5 \pm 11,3)$. En este estudio no se evaluaron estas variables porque todos los pacientes fueron tratados con ranura 0,018 y prescripción Roth.

No se relacionó la edad del paciente y los resultados del OGS en el estudio, parece ser que esta asociación es inexistente (Onyeaso \& BeGole, 2008). Por último no hubo diferencias en los puntajes del OGS entre pacientes con y sin extracciones lo cual está de acuerdo con lo descrito en la literatura (Vu et al.)

Brown reporta que realizar cambios curriculares y tener metas y objetivos terapéuticos claros dentro del posgrado, permite que los puntajes de OGS disminuyan progresivamente (Brown et al.) por lo tanto esta valoración de los puntajes del OGS son un insumo valioso para elaborar, optimizar y ejecutar planes de mejoramiento de la práctica clínica y para realizar futuras investigaciones en la Facultad de Odontología y en instituciones universitarias similares a la nuestra.
La insuficiente custodia de las ayudas diagnosticas de los pacientes limitó la posibilidad de utilizar un ID reconocido internacionalmente (Pulfer et al., 2009; Cangialosi et al.; American Board of Orthodontics). Este es un factor que limita las comparaciones con los resultados de otras instituciones.

En coclusión, el $80 \%$ de los pacientes terminados en el Posgrado de Ortodoncia de la Universidad de Antioquia tenían un grado de complejidad inicial entre comprometido o altamente comprometido. La evaluación del OGS varía entre 18 y 49 puntos (promedio $31,7 \pm 8,4$ ), solo 4 pacientes tuvieron 20 puntos o menos y el $50 \%$ pasaban los parámetros $\mathrm{ABO}$.

Además, el grado de complejidad inicial, afecta de manera determinante el resultado final del OGS; y aunque los valores de OGS encontrados son altos, son similares a los reportados por otras instituciones universitarias.

AGRADECIMIENTOS. AI Profesor Luis Gonzalo Álvarez por su colaboración y acompañamiento en el proceso estadístico.

BARBOSA, L. D.; . D.; ZAPATA, N. O.; CARVAJAL, A.; FRANCO, C. M.; RODRIGUEZ, A. S. A.; FLOREZ, P. A. A. \& RESTREPO, M. Outcome of orthodontic treatments and its relationship to malocclusion complexity. Int. J. Odontostomat., 8(2):201-206, 2014.

ABSTRACT: This descriptive study assessed the clinical static results of the treatment finished in the orthodontic graduate clinics of the University of Antioquia during the years 2010-2011, and its relationships with initial malocclusion complexity and some administrative factors. The records and complete medical histories of the patients were reviewed to determine the degree of initial malocclusion commitment and the final panoramic radiography and plasters were evaluated applying the Objective Grading System (OGS) proposed by the American Board of Orthodontic (ABO). Of 99 patients who completed treatment, 40 met the inclusion criteria. $80 \%$ of patients, who completed treatment, started with committed or highly committed malocclusion. The ABO OGS score found an average of 31.7 $( \pm 8.5)$ and $50 \%$ of the cases approved OGS criteria. The duration of treatment ( $55 \pm 22.25$ months) and the average number of appointments to complete treatment (37.3 111.4$)$ increased with the complexity of the initial malocclusion. The degree of initial malocclusion had statistically significant relationship with OGS scores $(p=0.018)$, while other administrative and clinical variables did not affect the results. The final values of OGS found are not ideal and are affected by the complexity of the initial malocclusion.

KEY WORDS: quality control, outcome assessment, malocclusion, index of orthodontic treatment need. 


\section{REFERENCIAS BIBLIOGRÁFICAS}

Ackerman, J. L. \& Proffit, W. R. The characteristics of malocclusion: a modern approach to classification and diagnosis. Am. J. Orthod., 56(5):443-54,1969.

Alford, T. J.; Roberts, W. E.; Hartsfield, J. K. Jr.; Eckert, G. J. \& Snyder, R. J. Clinical outcomes for patients finished with the SureSmile ${ }^{T M}$ method compared with conventional fixed orthodontic therapy. Angle Orthod., 81(3):383-8, 2011

American Board of Orthodontics. ABO Discrepancy Index Instructions Version 2009-2010. 2009. Disponible en: http:// www.americanboardortho.com/ professionals/downloads/ Discrepancy $\% 20$ Index $\% 20$ Scoring $\% 20$ Instructions.pdf

Asociación Médica Mundial. Declaración de Helsinki de la AMM Principios éticos para las investigaciones médicas en seres humanos. 2012. Disponible en http://www.wma.net/es/ 30publications/10policies/b3/

Brown, P. N.; Kulbersh, R. \& Kaczynski, R. Clinical outcomes assessment of consecutively finished patients in a 24-month orthodontic residency: a 5-year perspective. Am. J. Orthod. Dentofacial Orthop., 139(5):665-8, 2011.

Cameron, D. L. The relationship between American Board of orthodontics pretreatment dental cast discrepancy index scores and posttreatment dental cast objective grading system scores. Thesis. Saint Louis, Saint Louis University, 2010.

Campbell, C. L.; Roberts, W. E.; Hartsfield, J. K. Jr. \& Qi ,R. Treatment outcomes in a graduate orthodontic clinic for cases defined by the American Board of Orthodontics malocclusion categories. Am. J. Orthod. Dentofacial Orthop., 132(6):822-9, 2007.

Cangialosi, T. J.; Riolo, M. L.; Owens, S. E. Jr.; Dykhouse, V. J.; Moffitt, A. H.; Grubb, J. E.; Greco, P. M.; English, J. D. \& James, R. D. The ABO discrepancy index: a measure of case complexity. Am. J. Orthod. Dentofacial Orthop., 125(3):270-8, 2004

Casko, J. S.; Vaden, J. L.; Kokich, V. G.; Damone, J.; James, R. D.; Cangialosi, T. J.; Riolo, M. L.; Owens, S. E. Jr. \& Bills, E. D. Objective grading system for dental casts and panoramic radiographs. American Board of Orthodontics. Am. J. Orthod. Dentophacial Orthop., 114(5):589-99, 1998.

Costalos, P. A.; Sarraf, K.; Cangialosi, T. J. \& Efstratiadis, S. Evaluation of the accuracy of digital model analysis for the American Board of Orthodontics objective grading system for dental casts. Am. J. Orthod. Dentofacial Orthop., 128(5):624-9, 2005.

Deguchi, T.; Honjo, T.; Fukunaga, T.; Miyawaki, S.; Roberts, W. E. \& Takano-Yamamoto, T. Clinical assessment of orthodontic outcomes with the peer assessment rating, discrepancy index, objective grading system, and comprehensive clinical assessment. Am. J. Orthod. Dentofacial Orthop., 127(4):434-43, 2005.

Detterline, D. A.; Isikbay, S. C.; Brizendine, E. J. \& Kula, K. S. Clinical outcomes of 0.018-inch and 0.022-inch bracket slot using the ABO objective grading system. Angle Orthod., 80(3):528-32, 2010.
Hincapié, R. A.; Carvajal, F. A.; Sánchez, U. L. A. \& Arias, R. O. Satisfacción de los usuarios frente a la calidad de la atención prestada en el programa de Odontología Integral del Adolescente y Ortodoncia. Rev. Fac. Odontol. Univ. Antioq., 15(1):5-11, 2001.

Hou, H. M.; Wong, R. W. K. \& Hägg, U. The uses of orthodontic study models in diagnosis and treatment planning. Hong Kong Dent. J., 3(2):107-15, 2006.

Onyeaso, C. O. \& BeGole, E. A. Associations between pretreatment age and treatment time with orthodontic treatment outcome: A comparison by means of two orthodontic indice. Hell. Orthod. Rev., 11(1):9-20, 2008.

Paredes, V.; Gandia, J. L. \& Cibrián, R. Digital diagnosis records in orthodontics. An overview. Med. Oral Patol.Oral Cir. Bucal., 11(1):E88-93, 2006.

Pinskaya, Y. B.; Hsieh, T. J.; Roberts, W. E. \& Hartsfield, J. K. Comprehensive clinical evaluation as an outcome assessment for a graduate orthodontics program. Am. J. Orthod. Dentofacial Orthop., 126(5):533-43, 2004.

Pulfer, R. M.; Drake, C. T.; Maupome, G.; Eckert, G. J. \& Roberts, W. $E$. The association of malocclusion complexity and orthodontic treatment outcomes. Angle Orthod., 79(3):468-72, 2009.

Ministerio de Salud, Republica de Colombia. Normas científicas, técnicas y administrativas para la investigación en salud. 1993. Disponible en: http://www.minsalud.gov.co/Normatividad/ RESOLUCION\%208430\%20DE\%201993.pdf

Saxe, A. K.; Louie, L. J. \& Mah, J. Efficiency and effectiveness of SureSmile. World J. Orthod., 11(1):16-22, 2010.

Stock, G. J.; McNamara, J. A. Jr. \& Baccetti, T. Efficacy of 2 finishing protocols in the quality of orthodontic treatment outcome. Am. J. Orthod. Dentofacial Orthop., 140(5):688-95, 2011.

Vu, C. Q.; Roberts, W. E.; Hartsfield, J. K. Jr. \& Ofner, S. Treatment complexity index for assessing the relationship of treatment duration and outcomes in a graduate orthodontics clinic. Am. J. Orthod. Dentofacial Orthop., 133(1):9.e1-13, 2008.

Yang-Powers, L. C.; Sadowsky, C.; Rosenstein, S. \& BeGole, E. A. Treatment outcome in a graduate orthodontic clinic using the American Board of Orthodontics grading system. Am. J. Orthod. Dentofacial Orthop., 122(5):451-5, 2002.

Dirección para Correspondencia:

Prof. Ms. Diana Barbosa Lis

Prof. Dr. Alvaro Carvajal

Universidad de Antioquia

Calle $70 n^{\circ} 52-21$

Medellín - COLOMBIA

Recibido : 26-08-2013

Aceptado: 02-05-2014

Email: dimabarbosa@gmail.com carvajalflorez@gmail.com 